\title{
Serum Alkaline Phosphatase Levels and Mortality of Chronic Hemodialysis Patients
}

\author{
Tetsuri Yamashita', Junichi Shizuku², Takashi Ohba², Takashi Kabaya², Kosaku Nitta ${ }^{1 *}$ \\ ${ }^{1}$ Department of Medicine, Kidney Center, Tokyo Women's Medical University, Tokyo, Japan; ${ }^{2}$ Minami Senju Hospital, Tokyo, \\ Japan. \\ Email: *knitta@kc.twmu.ac.jp
}

Received April 26 ${ }^{\text {th }}, 2011$; revised July $6^{\text {th }}, 2011$; accepted July $25^{\text {th }}, 2011$.

\begin{abstract}
Objective: Alkaline phosphatase (ALP) is considered a biomarker of high bone turnover in hemodialysis (HD) patients with secondary hyperparathyroidism. This study was conducted to determine whether high serum ALP levels are associated with increased all-cause mortality of HD patients. Patients and Methods: This was a retrospective cohort study conducted at a single center. The subjects were 195 patients on chronic HD therapy who were followed up for a 5 years, and relationships between their baseline data and outcomes were assessed statistically. The serum ALP level was used as the predictor, and the primary end point was all-cause mortality. Results: Based on the median serum ALP of 236 $I U / L$, the subjects were divided into a low-ALP group $(<236 \mathrm{IU} / \mathrm{l})$ and a high-ALP group $(\geq 236 \mathrm{IU} / \mathrm{l})$. The high-ALP group was older and had a longer dialysis vintage, lower serum phosphorus concentrations, and higher serum parathyroid hormone levels, and they also had lower serum albumin levels and higher C-reactive protein values. In a multivariate Cox model in which the baseline serum ALP levels were used adjusted for age, gender, HD vintage, comorbidity, bone metabolism parameters, and serum liver enzyme levels, each doubling of the serum ALP level was associated with a significant increase in the hazard of all-cause mortality (hazard ratio 10.70, 95\% CI 1.53 - 74.24). Conclusion: High baseline serum ALP levels are associated with increased mortality of HD patients, independent of bone metabolism parameters and serum liver enzyme levels. ALP is a potential target for the treatment of HD patients.
\end{abstract}

Keywords: Alkaline Phosphatase, Hemodialysis, Mortality, Secondary Hyperparathyroidism

\section{Introduction}

Hyperparathyroidism secondary to severe chronic kidney disease has been reported to be associated with abnormal mineral metabolism and a poor outcome, especially in hemodialysis (HD) patients [1]. High serum calcium $(\mathrm{Ca})$-phosphorus $(\mathrm{P})$ products and elevated serum parathyroid hormone $(\mathrm{PTH})$ levels are associated with vascular calcification [2]. Serum alkaline phosphatase (ALP) levels are commonly elevated in secondary hyperparathyroidism, and the increase in serum ALP levels is considered to be a biomarker of high bone turnover. However, there is increasing evidence that ALP can promote vascular calcification by degrading pyrophosphate, an inhibitor of vascular calcification [3]. Kalantar-Zadeh et al. recently demonstrated an increased risk of all-cause mortality associated with higher baseline and time-varying ALP levels in HD patients, without including adjustments for elevated serum $\mathrm{P}$ and $\mathrm{Ca}$ levels [4]. We therefore investigated whether higher serum ALP levels are associated with increased all-cause mortality in chronic HD patients at a single center in Japan.

\section{Patients and Methods}

\subsection{Study Population}

This was a retrospective cohort study conducted at a single center. The study protocol was based on a previous report by Beddhu et al. [5] and approved by the institutional review board. Informed consent to evaluate their serum ALP levels was obtained from 196 of the patients who were undergoing maintenance HD treatment at Minami Senju Hospital, and they served as the subjects of this study. Patients began to be enrolled in the study in July 2005, and their course was followed through December 2010. The subjects underwent stable regular hemodialysis with bicarbonate dialysate. Patients were followed up for 5 years after enrollment, and the relationships between their baseline data and outcomes were assessed statistically. The data and causes of death were 
obtained by reviewing the subjects' hospital records. The clinical status of all subjects was evaluated by performing routine clinical examinations before each regular HD session.

\subsection{Biochemical and Other Measurements}

Blood was drawn immediately before starting each dialysis session with the subject in a fasting state. Serum albumin, urea nitrogen, aspartate aminotransferase (AST), alanine aminotransferase (ALT), Ca, P, and C-reactive protein (CRP) were measured by routine laboratory methods. Serum ALP (normal range: $203.0 \pm 4.6 \mathrm{IU} / \mathrm{L}$ ) was measured by an enzyme-linked immunosorbent assay (SSL Co., Kanagawa, Japan). The mean of two measurements made at the time of enrollment were used for the analysis. Serum intact PTH (normal range: 10 to 65 $\mathrm{pg} / \mathrm{mL}$ ) was measured once at the time of enrollment by an immunoradiometric assay (Allegro, Nichols Institute, San Juan Capistrano, CA, USA).

Systolic blood pressure and diastolic blood pressure were measured with a mercury sphygmomanometer after the patient had rested in the supine position for 10 to 15 minutes, and the mean values for 1 month were used for the analysis. Mean blood pressure (MAP) was calculated.

\subsection{Statistical Analyses}

Data are expressed as means $\pm \mathrm{SD}$ or as the median values and range. The cohort was divided into a low-ALP group and a high-ALP group, at the median baseline value. Baseline differences between the low-ALP group and high-ALP group in continuous variable were tested using ANOVA and differences in categorical variables were tested by Fisher's exact test. All-cause deaths were the outcomes of interest in the analyses. The associations between serum ALP levels and mortality were examined in Cox models that employed baseline serum ALP values. Unadjusted associations were first examined in each model and then they were examined after adjustments for age, gender, dialysis vintage, baseline comorbidity (coronary artery disease, cerebrovascular disease, peripheral vascular disease, diabetes and liver disease), body mass index (BMI), hemoglobin concentration, and serum albumin, AST and ALT levels. Serum Ca, P, and intact PTH levels were then added to determine whether the associations between the serum ALP levels and clinical outcomes were independent of these parameters. The above analyses were repeated with the serum ALP level used as a continuous variable. Because the distribution of serum ALP levels was skewed, they were logarithmically transformed and divided by the log of 2. Survival curves were estimated by the Kaplan-Meier method and evaluated by using the log-rank test. Prognostic variables for survival were examined first by using the univariate Cox proportional hazards method, and variables that were found to be significant were forced into multivariate Cox proportional hazards models. $\mathrm{P}$ values less than 0.05 were considered statistically significant. All calculations were performed on a Windows personal computer with StatView 5 statistical software (SAS Institute Inc, Cary, $\mathrm{NC})$.

\section{Results}

The mean age of the subjects in our cohort was $62.1 \pm$ 12.3 years; $61 \%$ of the subjects were men, and $43 \%$ were diabetes. The mean HD vintage was $114.7 \pm 102.8$ months. The mean BMI was $21.4 \pm 3.4 \mathrm{~kg} / \mathrm{m}^{2}$, and the mean serum albumin level was $3.6 \pm 0.3 \mathrm{~g} / \mathrm{dL}$. The mean hemoglobin concentration was $10.6 \pm 6.0 \mathrm{~g} / \mathrm{dL}$.

As shown in Figure 1, the distribution of the serum ALP levels was skewed to the right. The median ALP value was $236 \mathrm{IU} / \mathrm{L}$, and the range was $100 \mathrm{IU} / \mathrm{L}$ to 878 IU/L. There were $68(35.0 \%)$ all-cause deaths over a follow-up period of 5.0 years.

Table 1 summarizes the clinical characteristics of the subjects in the low-ALP group and high-ALP group, their median baseline ALP value (236 IU/1). Older age (p $=0.0028)$ and longer HD vintage $(p=0.0304)$ were associated with higher serum ALP levels. However, there were no differences between the two groups in baseline prevalence of baseline comorbid conditions. The mean AST and ALT values in both groups was within the normal ranges, and no statistical difference in both groups. Lower serum albumin levels $(p=0.0193)$ and higher CRP levels $(\mathrm{p}=0.0080)$ were associated with high serum ALP levels. Abdominal echography confirmed that increased serum levels of ALP, AST and ALT were not associated with hepatic diseases.

Table 2 summarizes the bone and mineral metabolism parameters in the two ALP groups. High serum ALP

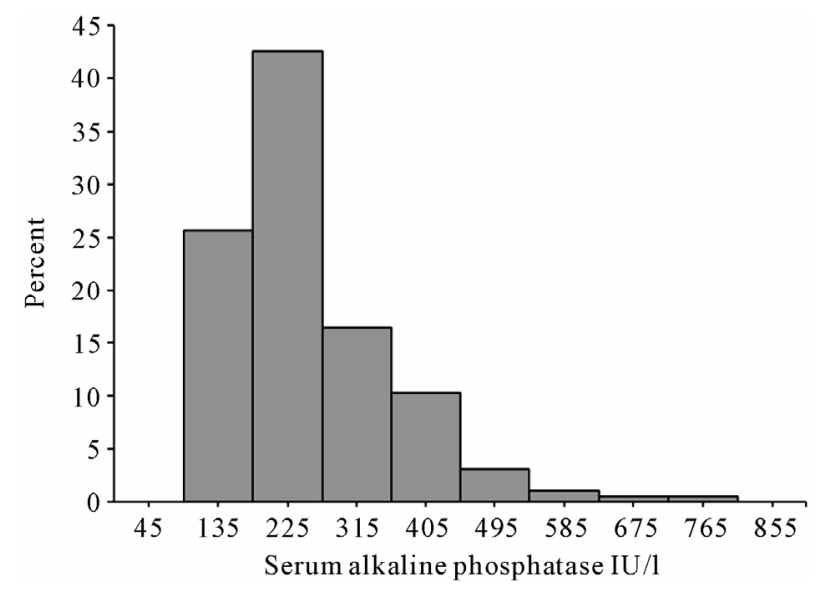

Figure 1. Distribution of the baseline serum alkaline phosphatase levels of chronic hemodialysis patients. 
Table 1. Patient characteristics according to their baseline alkaline phosphatase levels.

\begin{tabular}{|c|c|c|c|}
\hline & $\begin{array}{l}\text { Alkaline phosphatase }<236 \mathrm{IU} / 1 \\
(\text { mean } 176 \pm 34 \mathrm{IU} / 1) \mathrm{n}=98\end{array}$ & $\begin{array}{l}\text { Alkaline phosphatase } \geq 236 \mathrm{IU} / 1 \\
(\text { mean } 332 \pm 100 \mathrm{IU} / 1) \mathrm{n}=97\end{array}$ & $\mathrm{p}$ value* \\
\hline \multicolumn{4}{|l|}{ Demographics } \\
\hline Age, years & $59 \pm 12$ & $65 \pm 12$ & 0.0028 \\
\hline Male gender, $\%$ & 66 & 56 & 0.1434 \\
\hline \multicolumn{4}{|l|}{ Dialysis characteristics } \\
\hline Dialysis months & $98.9 \pm 95.1$ & $130.8 \pm 108.7$ & 0.0304 \\
\hline $\mathrm{Kt} / \mathrm{V}$ & $1.06 \pm 0.20$ & $1.07 \pm 0.20$ & 0.5897 \\
\hline \multicolumn{4}{|l|}{ Clinical characteristics } \\
\hline Diabetes, $\%$ & 48 & 39 & 0.1938 \\
\hline Cardio vascular disease, $\%$ & 14 & 8 & 0.2576 \\
\hline Cerebro vascular disease, $\%$ & 11 & 8 & 0.6303 \\
\hline Peripheral vascular disease, $\%$ & 2 & 4 & 0.4446 \\
\hline BMI, $\mathrm{kg} / \mathrm{m}^{2}$ & $21.7 \pm 3.7$ & $20.9 \pm 3.0$ & 0.0851 \\
\hline \multicolumn{4}{|l|}{ Laboratory data } \\
\hline Hemoglobin, $\mathrm{g} / \mathrm{dL}$ & $11.1 \pm 8.5$ & $10.2 \pm 0.9$ & 0.2766 \\
\hline Serum albumin, $\mathrm{g} / \mathrm{dL}$ & $3.7 \pm 0.3$ & $3.6 \pm 0.3$ & 0.0193 \\
\hline Aspartate aminotransferase, IU/L & $14.7 \pm 20.7$ & $14.9 \pm 8.5$ & 0.9287 \\
\hline Alanine aminotransferase, IU/L & $10.4 \pm 10.8$ & $11.2 \pm 9.4$ & 0.5760 \\
\hline $\mathrm{HCV}(+), \%$ & 16 & 15 & 0.9752 \\
\hline C-reactive protein, $\mathrm{mg} / \mathrm{dl}$ & $0.37 \pm 0.65$ & $0.84 \pm 1.55$ & 0.0080 \\
\hline Mean arterial pressure & $105.9 \pm 12.5$ & $103.9 \pm 15.4$ & 0.3243 \\
\hline \multicolumn{4}{|l|}{ Medication, $\%$} \\
\hline $\mathrm{CaCO}_{3}, \%$ & 88 & 78 & 0.0885 \\
\hline Sevelamer, \% & 13 & 11 & 0.8280 \\
\hline Vitamin D3, \% & 45 & 43 & 0.8856 \\
\hline
\end{tabular}

*p values calculated by ANOVA for continuous variables and Fisher's exact test for categorical variables.

levels were associated with lower serum $\mathrm{P}$ values $(\mathrm{p}=$ $0.0007)$ and higher intact PTH levels $(p=0.0043)$. The difference between the mean serum Ca level in low-ALP group and high-ALP group was not statistically different.

The results for the unadjusted associations between baseline serum ALP levels and all-cause mortality are shown in Figure 2. When expressed as a dichotomous factor, the high-ALP group was associated with increased mortality when compared with the low-ALP group ( $\mathrm{p}=$ $0.0074)$. When adjusted for age, gender, dialysis vintage, comorbidity (coronary artery disease, cerebrovascular disease, peripheral vascular disease, diabetes, liver disease), BMI, hemoglobin concentration, and serum albumin, AST and ALT levels, each doubling of baseline serum ALP was found to be associated with significant decrease in the hazard of all-cause mortality (Table 3). When also adjusted for the serum $\mathrm{Ca}, \mathrm{P}$, and intact PTH levels, the association between high baseline serum ALP levels and increased mortality increased even further (hazard ratio $10.70,95 \%$ CI 1.53 - 74.24).

\section{Discussion}

The results of this study demonstrated that the high-ALP group was significantly older and had a longer HD vintage. Higher baseline ALP levels were found to be associated with higher mortality than low baseline ALP levels 
Table 2. Baseline bone and mineral metabolism parameters according to their baseline serum alkaline phosphatase levels.

\begin{tabular}{|c|c|c|c|}
\hline & $\begin{array}{l}\text { Alkaline phosphatase }<236 \text { IU/1 } \\
(\text { mean } 176 \pm 34 \mathrm{IU} / 1) \mathrm{n}=98\end{array}$ & $\begin{array}{l}\text { Alkaline phosphatase } \geq 236 \mathrm{IU} / 1 \\
(\text { mean } 332 \pm 100 \mathrm{IU} / 1) \mathrm{n}=97\end{array}$ & $\mathrm{p}$ value \\
\hline Serum calcium $(\mathrm{mg} / \mathrm{dl})$ & $9.6 \pm 0.8$ & $9.4 \pm 1.0$ & 0.1825 \\
\hline Serum phosphorus (mg/dl) & $5.9 \pm 1.6$ & $5.2 \pm 1.4$ & 0.0007 \\
\hline Serum PTH (pg/ml) & $155 \pm 130$ & $244 \pm 245$ & 0.0043 \\
\hline Serum phosphorus $>5.5 \mathrm{mg} / \mathrm{dl}, \%$ & 60 & 36 & 0.0010 \\
\hline Serum calcium phosphorus product $(\mathrm{mg} / \mathrm{dl})^{2}$ & $57 \pm 16$ & $50 \pm 15$ & 0.0009 \\
\hline Serum calcium phosphorus product $\geq 55(\mathrm{mg} / \mathrm{dl})^{2}, \%$ & 53 & 32 & 0.0037 \\
\hline
\end{tabular}

*p values calculated by ANOVA for continuous variables and Fisher's exact test for categorical variables.

Table 3. Associations between baseline serum alkaline phosphatase levels and all-cause mortality.

\begin{tabular}{|c|c|c|}
\hline & \multicolumn{2}{|c|}{ Baseline serum alkaline phosphatase model } \\
\hline & Hazard ratio & $95 \% \mathrm{CI}$ \\
\hline \multicolumn{3}{|c|}{ Categorical variable models } \\
\hline \multicolumn{3}{|c|}{ High alkaline phosphatase group vs. low alkaline phosphatase (as reference) group } \\
\hline Unadjusted & 1.95 & $1.19-3.20$ \\
\hline Model 1* & 1.91 & $1.08-3.40$ \\
\hline Model $2 * *$ & 2.49 & $1.34-4.58$ \\
\hline \multicolumn{3}{|c|}{ Continuous variable models } \\
\hline \multicolumn{3}{|c|}{ For each doubling of serum alkaline phosphates } \\
\hline Unadjusted & 7.65 & $2.00-29.36$ \\
\hline Model 1* & 3.07 & $0.55-17.15$ \\
\hline Model $2 * *$ & 10.70 & $1.53-74.24$ \\
\hline
\end{tabular}

*Model 1 adjusted for demographics (age and gender), dialysis months, comorbidity (coronary artery disease, cerebrovascular disease, peripheral vascular disease, diabetes), BMI, hemoglobin, serum albumin, AST levels, **Model 2 further adjusted for serum calcium, phosphorus and PTH levels.

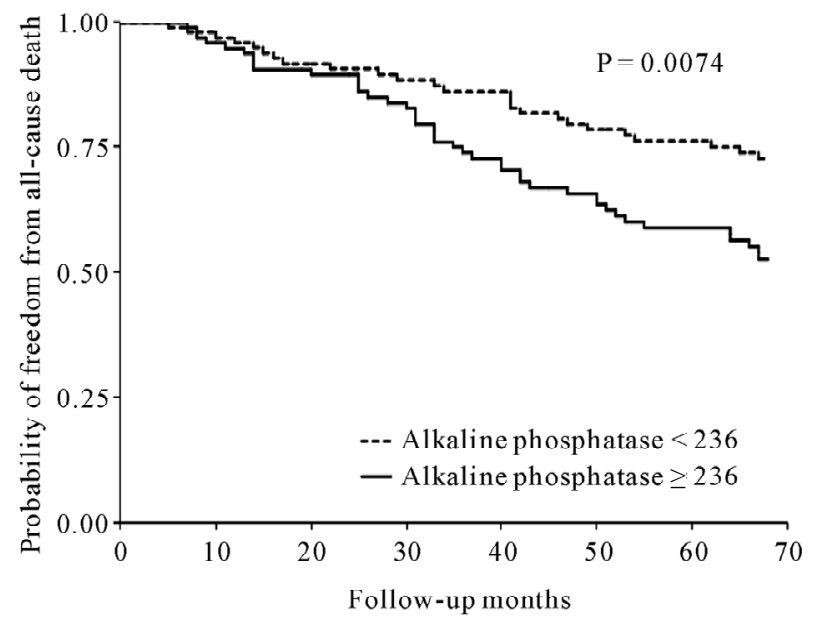

Figure 2. Kaplan-Meier plot of all-cause mortality in the low serum alkaline phosphatase group and the high serum alkaline phosphatase group. when the subjects were followed up for a period of 5 years. Moreover, the high ALP level was associated with lower serum P and higher intact PTH levels. These findings suggest that hyperparathyroidism is associated with higher mortality in chronic HD patients, and malnutrition and inflammation may be associated with increased mortality in HD patients with high serum ALP levels.

ALP is a hydrolase enzyme responsible for removing $\mathrm{P}$ from many types of molecules. ALP is produced by osteoblasts and plays an important role in bone mineralization by hydrolyzing pyrophosphate in the extracellular milieu [6]. Serum ALP is a biochemical marker of bone turnover and is used to monitor the metabolic bone disease associated with renal insufficiency [7]. Elevated serum ALP levels can be seen with worsening magnitude of bone turnover [8]. The magnitude of the enzyme elevation may indeed be a more reliable marker of severity of the high-turnover osteodystrophy than increased PTH 
levels, because the circulating serum ALP originates directly from the pathologic bone system.

It is likely that the association of ALP with mortality is related to vascular calcification. ALP has been shown in histological sections of vessels obtained from dialysis-associated calcific uremic arteriolopathy [9]. Indeed, genetic ablation of tissue-nonspecific ALP leads to amelioration of soft tissue calcification in animal studies [10]. ALP has been found to be up-regulated in calcified diabetic arteries [11], and the rate of pyrophosphate hydrolysis in the aorta was shown to be higher in uremic rats than in controls, and the increase was inhibited by levamisole, a non-specific inhibitor of ALP, suggesting a role for ALP in vascular calcification [3].

Elevated serum ALP levels were shown to be associated with progressive arterial calcification in a longitudinal study of 134 patients with Stage 4 or Stage 5 CKD, even after statistical adjustment for serum $\mathrm{Ca}, \mathrm{P}, \mathrm{PTH}$, fetuin A and CRP levels [12]. High serum ALP levels were also found to be associated with increased mortality of HD patients in an analysis of the DaVita database [4], but no adjustments for serum $\mathrm{Ca}$ and $\mathrm{P}$ levels were made in that analysis. A subsequent analysis of the Dialysis Outcomes and Practice Patterns Study (DOPPS) database after adjustment for serum $\mathrm{Ca}, \mathrm{P}$ and PTH levels found that elevated serum ALP levels in HD patients were associated with higher risks of hospitalization and death [13]. Data on liver enzymes (AST and ALT) are not available in the DOPPS database. A subsequent reanalysis of the DaVita database found that high ALP levels in HD patients were associated with increased mortality, after adjustment for serum $\mathrm{Ca}, \mathrm{P}, \mathrm{PTH}$ and AST levels [14].

Recently, Beddhu et al. [5] performed the longitudinal analyses of HEMO Study database to investigate the role of serum ALP on all-cause and mortality in HD patients. In a multivariate time-dependent Cox model using baseline and follow-up values of serum ALP levels, adjusted for demographics, HEMO Study groups, comorbidity, bone metabolism parameters and liver enzymes, each doubling of serum ALP was significantly associated with increased hazard of all-cause and cardiovascular mortality. Based on their study protocol, the current study was done independently in a different cohort. However, we reached to the similar conclusion that high baseline serum ALP levels were found to be associated with increased mortality of chronic HD patients independent of other bone metabolism parameters and liver enzymes, as previously published in the paper by Beddhu et al. [5].

Inflammation is another potential mechanism besides vascular calcification for the association between higher serum ALP levels and increased mortality. Serum ALP levels have been shown to be associated with elevated serum CRP levels $[15,16]$. Hypoalbuminemia has been found to be a strong risk factor for mortality among HD patients [17]. Hypoalbuminemia was presumed to result primarily from malnutrition. Zimmerman et al. found that serum CRP and albumin levels are independent predictors of all-cause mortality in HD patients [18]. Yeun et al. have reported that the acute-phase response or the cause of the acute-phase response is largely responsible for the effect of hypoalbuminemia on mortality in HD patients [19].

There was a significant association between high ALP levels and all-cause mortality in the baseline ALP model (Figure 2). When adjusted for age, gender, dialysis duration, comorbidity (coronary artery disease, cerebrovascular disease, peripheral vascular disease, diabetes, liver disease), BMI, hemoglobin concentration, and serum albumin, AST and ALT levels, each doubling of the baseline serum ALP levels was associated with significant decrease in the hazard of all-cause mortality (Table 3 ), and when also adjusted for the serum $\mathrm{Ca}, \mathrm{P}$ and intact PTH levels, the association between the baseline serum ALP and mortality increased. These findings suggest that high serum ALP levels are associated with increased mortality of HD patients, independent of bone metabolism parameters and liver enzyme levels.

Thus, there is increasing evidence that ALP may be more than a biomarker and an innocent bystander, and that it may play a pathogenic role in uremia leading to increased all-cause mortality. ALP may therefore serve as target for the treatment of HD patients, and novel ALP inhibitors that suppress vascular smooth muscle cell calcification have actually been identified [16]. The limitations of the study include the small cohort size and the fact that it was a retrospective study.

In summary, high baseline serum ALP levels were found to be associated with increased mortality of chronic HD patients independent of other bone metabolism parameters and liver enzymes. ALP may serve as a target for the treatment of HD patients.

\section{Acknowledgments}

The authors thank medical staffs of Minami Senju Hospital for collecting clinical data from medical records.

\section{REFERENCES}

[1] C. P. Kovesdy, S. Ahmadzadeh, J. E. Anderson and K. Kalantar-Zadeh, "Secondary Hyperparathyroidism is Associated with Higher Mortality in Men with Moderate to Severe Chronic Kidney Disease," Kidney International, Vol. 73, No. 11, 2008, pp. 1296-1302. doi:10.1038/ki.2008.64

[2] G. M. Chertow, P. Raggi, S. Chasan-Taber, J. Bommer, H. Holzer and S. K. Burke, "Determinants of Progressive 
Vascular Calcification in Haemodialysis Patients," $\mathrm{Ne}$ phrology Dialysis Transplantation, Vol. 19, No. 6, 2004, pp. 1489-1496. doi:10.1093/ndt/gfh125

[3] K. A. Lomashvili, P. Garg, S. Narisawa, J. L. Millan and W. C. O’Neill, "Upregulation of Alkaline Phosphatase and Pyrophosphate Hydrolysis: Potential Mechanism for Uremic Vascular Calcification," Kidney International, Vol. 73, No. 9, 2008, pp. 1024-1030. doi:10.1038/ki.2008.26

[4] K. Kalantar-Zadeh, N. Kuwae, D. L. Regidor, et al., "Survival Predictability of Time-Varying Indicators of Bone Disease in Maintenance Hemodialysis Patients," Kidney International, Vol. 70, No. 4, 2006, pp. 771-780. doi:10.1038/sj.ki.5001514

[5] S. Beddhu, B. Baird, X. Ma, A. K. Cheung and T. Greene, "Serum Alkaline Phosphatase and Mortality in Hemodialysis Patients," Clinical Nephrology, Vol. 74, No. 2, 2010, pp. 91-96.

[6] M. Schoppet and C. M. Shanahan, "Role for Alkaline Phosphatase as an Inducer of Vascular Calcification in Renal Failure?" Kidney International, Vol. 73, No. 9, 2008, pp. 989-991. doi:10.1038/ki.2008.104

[7] P. Magnusson, C. A. Sharp, M. Magnusson, J. Risteli, M. W. Davie and L. Larsson, "Effect of Chronic Renal Failure on Bone Turnover and Bone Alkaline Phosphatase Isoforms," Kidney International, Vol. 60, No. 1, 2001, pp. 257-265. doi:10.1046/j.1523-1755.2001.00794.x

[8] S. Fletcher, R. G. Jones, H. C. Rayner, et al., "Assessment of Renal Osteodystrophy in Dialysis Patients: Use of Bone Alkaline Phosphatase, Bone Mineral Density and Parathyroid Ultrasound in Comparison with Bone Histology," Nephron, Vol. 75, No. 4, 1997, pp. 412-419.

[9] M. Reslerova and S. M. Moe, "Vascular Calcification in Dialysis Patients: Pathogenesis and Consequences," American Journal of Kidney Disease, Vol. 41, No. 3, pp. S96-S99. doi:10.1053/ajkd.2003.50094

[10] S. Narisawa, D. Harmey, M. C. Yadav, W. C. O’Neill, M. F. Hoylaerts and J. L. Millan, "Novel Inhibitors of Alkaline Phosphatase Suppress Vascular Smooth Muscle Cell Calcification," Journal of Bone Mineral Research, Vol. 22, No. 11, 2007, pp. 1700-1710. doi:10.1359/jbmr.070714

[11] G. P. Fadini, P. Pauletto, A. Avogaro and M. Rattazzi, "The Good and the Bad in the Link between Insulin Re- sistance and Vascular Calcification," Atherosclerosis, Vol. 193, No. 2, 2007, pp. 241-244.

[12] M. K. Sigrist, M. W. Taal, P. Bungay and C. W. McIntyre, "Progressive Vascular Calcification over 2 Years Is Associated with Arterial Stiffening and Increased Mortality in Patients with Stages 4 and 5 Chronic Kidney Disease," Clinical Journal of American Society of Nephrology, Vol. 2, No. 6, 2007, pp. 1241-1248. doi:10.2215/CJN.02190507

[13] M. J. Blayney, R. L. Pisoni, J. L. Bragg-Gresham, et al., "High Alkaline Phosphatase Levels in Hemodialysis Patients are Associated with Higher Risk of Hospitalization and Death," Kidney International, Vol. 74, No. 5, 2008, pp. 655-663. doi:10.1038/ki.2008.248

[14] D. L. Regidor, C. P. Kovesdy, R. Mehrotra R, et al., "Serum Alkaline Phosphatase Predicts Mortality among Maintenance Hemodialysis Patients," Journal of American Society of Nephrology, Vol. 19, No. 11, 2008, pp. 2193-2203. doi:10.1681/ASN.2008010014

[15] A. Kerner, O. Avizohar, R. Sella, et al., "Association between Elevated Liver Enzymes and C-reactive Protein: Possible Hepatic Contribution to Systemic Inflammation in the Metabolic Syndrome," Arteriosclerosis Thrombosis Vascular Biology, Vol. 25, No. 1, 2005, pp. 193-197.

[16] B. M. Cheung, K. L. Ong, R. V. Cheung, et al., "Association between Elevated Liver Enzymes and C-reactive Protein in Hong Kong Chinese," Clinical Chemistry and Laboratory Medicine, Vol. 46, No. 4, 2008, pp. 523-527. doi:10.1515/CCLM.2008.111

[17] E. G. Lowrie and N. L. Lew, "Death Risk in Hemodialysis Patients: The Predictive Value of Commonly Measured Variables and an Evaluation of Death Rate Differences between Facilities," American Journal of Kidney Disease, Vol. 15, No. 5, 1990, pp. 458-482.

[18] J. Zimmermann, S. Herrlinger, A. Pruy, T. Metzger and C. Wanner, "Inflammation Enhances Cardiovascular Risk and Mortality in Hemodialysis Patients," Kidney Internation, Vol. 55, No. 2, 1999, pp. 648-658.

[19] J. Y. Yeun, R. A. Levine, V. Mantadilok and G. A. Kaysen, "C-Reactive Protein Predicts All Cause and Cardiovascular Mortality in Hemodialysis Patients," American Journal Kidney Disease, Vol. 35, No. 3, 2000, pp. 469476. doi:10.1016/S0272-6386(00)70200-9 